\title{
Psychiatric Complications of Obesity and Its Treatment: A Brief Review
}

\author{
Ghanem Al Hassani' ${ }^{1}$ Amir A. Mufaddel2* \\ ${ }^{1} \mathrm{Al}$ Ain Hospital, Al Ain, UAE \\ ${ }^{2}$ Department of Internal Medicine, Division of Psychiatry, Tawam Hospital, Al Ain, UAE \\ Email: *khalifaamir@yahoo.co.uk
}

How to cite this paper: Al Hassani, G. and Mufaddel, A.A. (2019) Psychiatric Complications of Obesity and Its Treatment: A Brief Review. Open Journal of Psychiatry, 9, 267-280.

https://doi.org/10.4236/ojpsych.2019.94020

Received: September 23, 2019

Accepted: October 20, 2019

Published: October 23, 2019

Copyright $\odot 2019$ by author(s) and Scientific Research Publishing Inc. This work is licensed under the Creative Commons Attribution International License (CC BY 4.0).

http://creativecommons.org/licenses/by/4.0/

(c) (i) Open Access

\begin{abstract}
Obesity-defined as a body mass index (BMI) of $30 \mathrm{~kg} / \mathrm{m}^{2}$ or more-is associated with a wide range of medical and psychiatric complications. Physical co-morbidities include: insulin resistance, type 2 diabetes mellitus, dyslipidemia, asthma, hypertension, coronary heart disease, osteoarthritis of the knee and polycystic ovary syndrome. Mental health problems are particularly more common among individuals presenting for treatment of obesity than those in the community. They have higher rates of depression, anxiety, increased substance abuse and an increased prevalence in eating disorders. It has been estimated that up to $60 \%$ of extremely obese individuals have been suffering from an Axis I psychiatric disorder; most commonly mood and anxiety disorders. Obstructive sleep apnea (OSA) can occur with obesity and can also lead to sleep disorders and psychiatric complications. Up to $40 \%$ of patients with sleep apnea were found to have affective disorder or alcohol abuse. Weight loss medications and surgery may add to the problem of psychological complications of obesity. For example, in 2008, rimonabant development was discontinued by manufacturer as it has been associated with an increased risk of adverse psychiatric events including suicidal ideation and suicidal behavior. On the other hand, the prevalence of obesity is also high among patents with psychiatric illness, which can be caused by the effects of psychotropic medications which can lead to increased appetite, weight gain, sedation and psychomotor retardation. Obesity is reported in more than $60 \%$ of patients with schizophrenia and bipolar disorder. Food intake is regulated by several neurotransmitters, peptides and amino acids. Antipsychotics which block dopamine D2 receptors increase appetite and result in significant weight gain, while drugs that increase brain dopamine concentration are anorexigenic.
\end{abstract}




\section{Keywords}

Obesity, Psychiatric Complications, Anxiety, Depression, Mood Disorder, Sleep Apnea, Bariatric Surgery, Antipsychotics

\section{Introduction}

Obesity is defined as a body mass index (BMI) of $30 \mathrm{~kg} / \mathrm{m}^{2}$ or more; while overweight individuals are defined as having BMI between $25 \mathrm{~kg} / \mathrm{m}^{2}$ and $29.9 \mathrm{~kg} / \mathrm{m}^{2}$. Morbid/severe obesity is defined as a BMI of $40 \mathrm{~kg} / \mathrm{m}^{2}$ or higher or a BMI of 35 $\mathrm{kg} / \mathrm{m}^{2}$ along with one of the comorbidities (including: cardiovascular disease, type II diabetes, hypertension, respiratory disorders, osteoarthritis, infertility and reproductive disorders, and cancer) [1] [2]. Prevalence of obesity has been estimated ranging between $15 \%$ and $20 \%$ in industrialized countries [3]. Higher prevalence estimates of obesity were found in the United States approaching $30.4 \%$ for obese adults and 16\% for children/adolescents [4]. Extreme obesity is more common in women (6.9\%) than men (2.8\%) in the United States [5].

Lipids have multiple specialized roles in neuronal function such as conduction of electrical impulses, synaptic function, and complex signaling pathways [6]. Several neurotransmitters (such as dopamine, GABA, norepinephrine, serotonin), peptides and amino acids are involved in the regulation of food intake. Dopamine seems to regulate food intake by modulating food reward via the mesolimbic circuitry of the brain. Drugs which block dopamine D2 receptors increase appetite and result in significant weight gain (e.g. antipsychotic medications), while drugs that increase brain dopamine concentration are anorexigenic [7]. The genetic molecules that modulate hypothalamic activity (i.e. leptin, ghrelin, insulin) are also expressed in limbic brain regions involved with reward, motivation, learning, emotion, and stress responses and they are likely to modulate food consumption [8]. Ward and colleagues found that elevated BMI is associated with reduced brain volumes. Episodic learning, working memory, and processing speed abilities were not associated with BMI in their sample indicating that the possible effect on cerebral atrophy had not influenced cognition in participants with a high BMI [9]. In a 24-year follow-up study of body mass index and cerebral atrophy, D. Gustafson and colleges found significant risk of temporal atrophy associated with increase in BMI [10].

\section{Obesity and Psychiatric Co-Morbidities}

The definition of co-morbidity includes any condition associated with obesity that usually worsens as the degree of obesity increases and that often improves as the condition is treated [11]. Obesity is associated with both physical \& psychosocial complications. Physical co-morbidities include: insulin resistance, type 2 diabetes mellitus, dyslipidemia, asthma, hypertension, coronary heart disease, osteoarthritis of the knee and polycystic ovary syndrome. According to the world 
health organization (WHO), up to $20 \%$ of the developed countries population may suffer from obesity-related hypertension and in most population studies, about $85 \%$ - 95\% of patients with type-II diabetes are obese [12].

Regarding psychiatric comorbidity; obese individuals in general do not report more mental health problems than normal-weight individuals; but those who are seeking treatment in commercial weight-loss programs or obesity clinics were reported to have higher rates of depression, increased substance abuse and an increased prevalence in eating disorders, particularly binge eating disorder and night eating syndrome [13]. Up to $60 \%$ of extremely obese individuals have been suffering from an Axis I psychiatric disorder; most commonly mood and anxiety disorders [14]. It has been well established that psychopathology is more reported by individuals presenting for treatment of obesity than those in the community. For example, the psychiatric comorbidities in candidates for weight loss surgery in one study were; mood disorder (15.6\%), anxiety disorder (24\%), substance disorder (1.7\%), eating disorder (16.4\%) and personality disorder (28.9\%) [15]. Weight loss medications and even surgery may add to the problem of psychological complications of obesity. In 2008, rimonabant development was discontinued by manufacturer as it has been associated with an increased risk of adverse psychiatric events including suicidal ideation and suicidal behavior [16] [17].

\subsection{Prevalence of Obesity among Psychiatric Populations}

On the other hand, there is high prevalence of obesity among mentally ill population, which may be partly caused by the effects of psychotropic medications due to side effects including increased appetite, weight gain, sedation and psychomotor retardation. A recent study of patients with schizophrenia and bipolar disorder has found that $63 \%$ of participants with schizophrenia and $68 \%$ of those with bipolar disorder were obese; and the obesity in this study was more likely to be associated with female gender, treatment with mood stabilizers and taking a greater number of psychiatric medications [18].

In a nationally representative sample of US adults including 9125 respondents found obesity to be associated with an approximately $25 \%$ increase in odds of mood and anxiety disorders and an approximately $25 \%$ decrease in odds of substance use disorders with no variation between males and females with regard to association between psychiatric disorders and obesity in this study [19].

Obese individuals have high prevelance of drug dependence and personality disorders [15] [19]. Positron Emission Tomography (PET) studies indicated that obese individuals have significantly lower dopamine D2 receptor levels, which is similar to findings from PET studies in drug-addicted subjects which may be explained by the reward circuits that mediating both obesity and drug addiction [20].

\subsection{Depression}

Studies have identified a relationship between depression, suicidality, and obesi- 
ty which makes ensuring psychiatric assessment and proper psychiatric treatment important elements in the management of obesity including candidates for bariatric surgery [21]. The association between obesity and depression may depend on the severity of obesity. Studies comparing obese individuals with normal-weight parsons, reported that obese subjects have an approximately 1.5 -fold higher prevalence of past-month depression than their normal-weight counterparts [22]. A large epidemiologic study including over 40,000 US adults found the risk of major depression in persons with a BMI of 40 or greater was nearly 5 times that in persons of average weight. The results of the study indicated a U-shaped relationship that relatively high and low BMI values were found to be associated with an increased probability of being diagnosed major depression. The study suggested gender differences as the results indicated about $37 \%$ increased probability of major depression diagnosis in female obese participants, while males had a decreased risk of depression of similar magnitude. Similar relationships were found between BMI values and suicidal ideations. Among females, a 10-unit increase in BMI increased the risk of past year suicide ideation and attempt by $22 \%$ while in males the past year suicide ideation and attempt reduced the risk of past year suicide ideation and attempt by $26 \%$ and $55 \%$ respectively. Compared to men of average weight, those men who were under-weight were more likely to have suicidal ideations, suicidal attempts and clinical depression ( $81 \%, 77 \%$ and $25 \%$ respectively) [23].

A recent systematic review and meta-analysis was conducted on the longitudinal relationship between depression, overweight and obesity including $15 \mathrm{stu}$ dies. The meta-analysis has confirmed the reciprocal relationship between obesity and depression. Obesity was found to increase the risk of depression; and depression increased the odd ratios for developing obesity [24].

Dixon and colleagues documented significant comorbidity between severe obesity and depression using Beck Depressive Inventory (BDI) which indicated that the majority of patients (53\%) scored 16 or greater depressive symptoms which were strongly associated with poor quality of life scores. Those findings have no association with measures of weight distribution, fasting insulin level, insulin resistance or diseases associated with the metabolic syndrome (e.g. diabetes and hypertension) [11].

Studies have documented significant depressive symptoms in treatment-seeking obese adolescents compared with community-based obese or non-obese control groups. One of these studies investigated depressive symptoms in extremely obese adolescents presenting for bariatric surgery reported approximately $30 \%$ showing depressive symptoms within a clinical range using the (BDI)-II [25]. Some studies found no significant difference between normal-weight and obese adolescence; in community samples of adolescents, regardless of gender, socioeconomic status or ethnicity, reports of depressive symptoms are not significantly higher in obese than normal-weight groups [26]. However; treatment of depression may allow patients to successfully address lifestyle changes [13]. 


\subsection{Anxiety Disorders}

Anxiety disorders are common in obese individuals and seem to be particularly common among females and those suffering from binge eating disorder. Data from a Canadian community health survey indicated that the prevalence of anxiety disorders in obese individuals (3.0\%) is higher than anxiety in individuals with adequate weight (2.6\%). The study also reported that $3.4 \%$ of obese individuals had been diagnosed with both mood and anxiety disorders, compared with $1.9 \%$ among individuals with adequate weight [27]. Obese Individuals with binge eating disorder have high level of anxiety which was estimated to be $41 \%$ in a consecutive series of 142 participants [28]. An Australian community survey indicated that obesity in females was associated with more anxiety and depressive symptoms, but there were weak and inconsistent associations in men [29]. Most of the studies showed a positive relationship with panic disorder, specific and social phobia, and generalized anxiety disorder. Less commonly, obesity may also be associated with other types of anxiety such as agoraphobia, panic attacks and posttraumatic stress disorder [30].

\subsection{Sleep Disorders and Obesity}

Obstructive sleep apnea (OSA) may be associated with obesity and may also be associated with depression. OSA is defined by the presence of excessive daytime somnolence and a polysomnogram apnea-hyperpnoea index above 5 during sleep hours [31]. It is characterized by recurrent episodes of complete (apnea) or partial obstruction (hypopnea) of the upper airway during sleep associated with progressive respiratory effort to overcome the obstruction. These obstructive respiratory events are associated with cortical micro-arousals and oxygen desaturation which lead to sleep fragmentation, chronic sleep loss, and increased sympathetic neural activity [32]. Insomnia and metabolic complications of OSA may be related to hypothalamic-pituitary-adrenal axis hyperactivity [33]. OSA induces alveolar hypoventilation and respiratory insufficiency, and contributes to other cardiopulmonary consequences such as pulmonary hypertension and corpulmonale, and therefore, increases the burden of clinically significant obesity [34] [35]. The prevalence of OSA in severe obesity is very high ranging between $50 \%$ and $98 \%$. Up to $50 \%$ of severely obese subject commonly complain of fatigue, daytime sleepiness and non-refreshing sleep despite the absence of sleep-disordered breathing [32]. The incidence of OSA has been under-estimated in patients who present with morbid obesity for evaluation for weight loss although obese and over-weight individuals are at the highest risk for developing OSA [36]. The study done by Hallowell and colleagues supported the idea that OSA is under-diagnosed in morbidly obese patients; they found only $19 \%$ of patients presenting for bariatric surgery during the study period had a diagnosis of OSA, which increased to 56\% when clinical parameters and the Epworth Sleep Score were used; and when all patients were evaluated by a polysomnogram, the diagnosis of OSA increased to $91 \%$ [37]. 
Depression has been reported frequently in association with sleep apnea syndromes. About $40 \%$ of patients with sleeps apnea were found to have affective disorder or alcohol abuse [38].

There might be improvement in OSA following bariatric surgery; particularly gastric bypass [39]. Leandro G. Fritscher et al. found correlation between weight loss following bariatric surgery for morbid obesity and improvement in mean oxygen saturation associated with significant long-term improvements in obstructive respiratory event, oxygenation and resolution of daytime somnolence [40].

Four studies have reported changes in sleep pattern assessed by polysomnography before and after surgically induced weight loss. There were significant reductions in BMI and arousal index [35].

\subsection{Eating Behavior \& Binge Eating Disorder}

Eating behavior can be predictor of adult weight gain. Commonly assessed eating behaviors are disinhibition, restraint, and hunger [41]. The most common condition is binge eating disorder, which is characterized by eating a large amount of food within a 2-hour period associated with feeling of a subjective loss of control. Regarding the relation between eating disorders and bariatric surgery, growing body of evidence suggests the occurrence of postoperative binge eating to be associated with weight regain in the first two years following surgery [42]. Binge eating disorder and night eating syndrome are important factors in obese individuals presenting for bariatric surgery for two reasons: their prevalence rates have been reported to be higher than in non-surgery-seeking samples, and the possibility that eating disorders may influence weight loss after surgery [26].

\subsection{Stigma and Impact on Quality of Life (QOL)}

Obese individuals are perceived by others as being ugly, stupid, mean, sloppy, lazy, dishonest, worried, sad, self-indulgent, and unlikable and emotionally impaired [43]. The pervasive and deleterious effects of weight based stigmatization have been documented in research since the 1960s [44]. Overt hostility toward and bias against, obese individuals have been found among different professions and situations including: social, educational, occupational and medical settings [45]. Internalization of stigma of the society's negative views of obesity has been suggested because of the obese individuals whom found to display weight-related bias [46].

Quality of life is defined as "the perception of the individual in life, in the context of culture and in the system of values where he/she lives in relation to his/her objectives, expectations, standards and concerns" [47]. Considerable literature documented a consistent and strong relationship between obesity in pediatric patients and impaired health related quality of life (HRQOL) affecting physical, social \& emotional functioning [48]. Both self-reported and parent-proxy 
HRQoL were found to be markedly impaired and significantly lower relative to norms on healthy youth. A retrospective review including 33 extremely obese adolescents presenting for bariatric surgery has shown thatthe mean score for total self-reported HRQoL was 54.9 for extremely obese adolescents compared with 83.0 for healthy adolescents. Similarly, the mean score for total parent-proxy HRQoL was 48.4 for extremely obese adolescents compared with 87.6 for healthy adolescents [49].

\section{Psychosocial Outcome of Obesity Treatment}

Long-term efficacy and effectiveness of obesity treatments is poor which may be due to barriers that undermine long-term strategies for obesity management including lack of recognition of obesity as a chronic condition, low socioeconomic status, time constraints, intimate saboteurs, and a wide range of comorbidities including mental health, sleep, chronic pain, musculoskeletal, cardiovascular, respiratory, digestive and endocrine disorders [13].

\subsection{Bariatric Surgery}

Because of the limited success of non-surgical interventions in the management of severe obesity; a National Institute of Health (NIH) Consensus Panel recommended bariatric surgery for well-informed, motivated obese patients with BMIs of 40 0r higher or BMIs 35 - 40 with significant obesity-related comorbidity who have acceptable risks for operation. Pre-surgical multidisciplinary evaluation of bariatric surgery candidates is recommended including pre-surgical psychiatric evaluation due to documented high rates of psychiatric disorders among bariatric surgery candidates [1] [39].

A recent systemic review and meta-analysis found bariatric surgery to be effective for weight loss achievement with a substantial complete resolution or improvement of diabetes, hyperlipidemia, hypertension and obstructive sleep apnea [39]. Weight loss following bariatric surgery is associated with significant improvements in many obesity-related comorbidities and psychosocial status postoperatively including self-esteem, depressive symptoms, quality of life and body image which improve in the first year after surgery [43]. Psychosocial changes occur following bariatric surgery, in both positive \& negative terms [50]. Bariatric surgery has significant long-term effects on body weight, obesity-related comorbidities and quality of life [51]. Improvement in depressive symptoms has been reported following significant weight loss after bariatric surgery and behavioral and dietary interventions [11].

After successful bariatric surgery; there is reported increase in the perception of wellbeing, social function, body self-image, self-confidence, ability to interact with others and time spent in recreational and physical activities [39]. Villela and colleagues evaluated the degree of QOL in patients with morbid obesity before and after bariatric surgery; they found statistically significant improvement in QOL detected in the aspects of general health, functional ability and vitality 
[47]. A controlled cross sectional study, comparing clinically obese patients waiting for surgery with three different groups of patients at three different postsurgical times, found that the first year after gastric bypass surgery showed major life changes. There were significant changes by 2 to 4 weeks post-surgery evident in subject's appraisal of their overall health and self-esteem. They also found that improvements in BMI, depression, self-esteem, and HRQL do not seem to parallel each other in a linear fashion [52].

Busetto et al. compared weight regains after 4 years of follow-up in binge eaters and non-binge eaters and was found to be similar in both groups [53]. A recent review of the literature addressing binge eating in the bariatric surgery patients revealed no consistent outcomes in relationship between pre-surgical binge eating and post-surgical weight loss across studies. Binge eating behavior following surgery is associated with poorer weight outcomes; and binge eating behavior existing prior to surgery should be considered a warning sign for potentially less successful weight loss outcomes [54].

Modest weight reductions (5\% - 10\% in initial weight) are associated with significant health benefits for overweight \& obese individuals [42]. Improvement in physical comorbidities and quality of life may play a role in improvement of psychiatric comorbidity. Anderson JR et al. compared symptoms of anxiety and depression before and after duodenal switch; they found statistically significant sustained reductions in the symptoms of anxiety and depression after the duodenal switch procedure which were closely associated with improvements in self-reported physical health [55].

Neuropsychiatric complications following gastric bypass surgery have been reported in the medicalliterature, such as severe psychotic disorders, Wernicke's encephalopathy, and Korsakoff's syndrome [56]. Bond and colleagues found increased levels of depressive symptoms among successful weight losers in both groups of surgical and nonsurgical methods from study entry to one year follow-up [57].

\subsection{Pharmacological Treatment of Obesity}

Currently, there are no effective medications for treatment of obesity especially morbid obesity [1]. Weight loss medications can be divided into two categories: appetite suppressants and lipase inhibitors based on their mechanisms of action. Pharmacologic therapy can be offered to patients who have failed to achieve weight loss through diet and exercise alone. For adjunctive drug therapy, options include sibutramine, orlistat, phentermine, diethylpropion, fluoxetine, and bupropion. The choice of agent will depend on the side effects profile of each drug and the patient's tolerance of those side effects [58].

A meta-analysis which assessed the efficacy and safety of weight loss medications approved by the US Food and Drug Administration and other medications that have been used for weight loss. The meta-analysis concluded that sibutramine, orlistat, phentermine, probably diethylpropion, bupropion, probably flu- 
oxetine, and topiramate promote modest weight loss when given along with recommendations for diet. Sibutramine and orlistat are the 2 most-studied drugs [58] [59].

For patients suffering from comorbid obesity and depression or anxiety; use of psychotropic medications that may serve both goals may be a good choice such as the selective serotonin re-uptake inhibitors fluoxetine and sertraline. There are 9 studies of fluoxetine treatment with reported weight loss outcomes with doses higher than those used for depression; while there was one small study for sertraline with no significant findings. There were 3 articles for a pooled analysis of the efficacy of bupropion for weight loss; two of them included diet as a co-intervention, and 1 study included exercise. The pooled weight loss in the bupropion-treated patients was $2.77 \mathrm{~kg}$ at 6 to 12 months [58] [59].

\subsection{Behavioral Interventions \& Exercise}

Behavioral interventions for management of obesity include exercise, decreased calorie intake and cognitive behavioral approaches. Trials of exercise and dietary restriction to modify body occurred when work has become increasingly sedentary [60].

Behavior therapy is both the most studied and most effective therapy for treating obesity at present [61]. Diettherapiesare is relatively ineffective in management of severe obesity in the long term [39]. Weight loss resulting from dieting is often associated with improvements in self-esteem, mood and body image [46].

Behavioral interventions are recommended for psychiatric patients who are suffering from obesity secondary to antipsychotic treatment. A large meta-analysis showed that prevention of weight gain at treatment onset is more beneficial than weight reduction after initiation of treatment in terms of acceptability \& cost effectiveness [62]. Cognitive behavior therapy can be provided by registered psychologists and can also be provided by other trained health professionals such as physicians, dieticians, exercise physiologists or psychiatrists [63].

Exercise is needed to be incorporated into every strategy intended to prevent weight gain as well as to maintain weight loss over time. Evidence is accumulating the profound benefits of exercise for brain function, including improvements in learning and memory as well as in preventing and delaying loss of cognitive function with aging or neurodegenerative disease [64].

A recent meta-analysis found that lifestyle modification was superior to control and non-dieting interventions for reducing symptoms of depression, and marginally better than dietary counseling and exercise-alone programs. Exercise-alone programs were superior to controls [65]. Improvement in mood symptoms with exercise seems to be related to the dose and frequency of exercise; a 16 week exercise intervention was shown to be as effective as antidepressants in treatment of older depressed individuals; a recent study concluded that 
although 30 min of either moderate-intensity treadmill exercise or quiet rest is sufficient to improve the mood and well-being of patients with major depressive disorder, exercise appears to have a greater effect on the positively valence states measured. Moderate exercise dose has therapeutic effect on major depression but low dose exercise has not [66]. There is little research supporting the theories explaining the mechanisms for the exercise-depression relationship; these theories include several physiologic and psychological mechanisms such as the endorphin hypothesis, the thermogenic hypothesis, the monoamine hypothesis, the distraction hypothesis and the enhancement of self-efficacy [67].

\section{Conclusion}

Obesity is a health problem with profound impact on both physical and psychological health. Comorbid psychosocial problems include depression, anxiety, stigma, poor QOL, and sleep problems. Treatment strategies for obese individuals suffering from associated depression or anxiety may include cognitive behavioral approaches, exercise and SSRIs (fluoxetine) which have shown benefits in improving both mood and obesity.

\section{Conflicts of Interest}

The authors declare no conflicts of interest regarding the publication of this paper.

\section{References}

[1] Marcus, M.D., Kalarchian, M.A. and Courcoulas, A.P. (2009) Psychiatric Evaluation and Follow-Up of Bariatric Surgery Patients. American Journal of Psychiatry, 166, 285-291. https://doi.org/10.1176/appi.ajp.2008.08091327

[2] Bennet, J.M., Mehta, S. and Rhodes, M. (2007) Surgery for Morbid Obesity. Postgraduate Medical Journal, 83, 8-15. https://doi.org/10.1136/pgmj.2006.048868

[3] Patrick, D.L., Bushnell, D.M. and Rothman, M. (2004) Performance of Two Self-Report Measures for Evaluating Obesity and Weigh Loss. Obesity Research, 12, 48-57. https://doi.org/10.1038/oby.2004.8

[4] Hedley, A.A., Ogden, C.L., Johnson, C.L., et al. (2004) Prevalence of Overweight and Obesity among US Children, Adolescents, and Adults, 1999-2002. The Journal of the American Medical Association, 291, 2847-2850. https://doi.org/10.1001/jama.291.23.2847

[5] Ogden, C.L., Carroll, M.D., Curtin, L.R., et al. (2006) Prevalence of Overweight and Obesity in the United States. The Journal of the American Medical Association, 295, 1549-1555. https://doi.org/10.1001/jama.295.13.1549

[6] Gross, R.W., Jenkins, C.M., Yang, J., Mancuso, D.J. and Han, X. (2005) Functional Lipidomics: The Role of Specialized Lipids and Lipid-Protein Interactions in Modulating Neuronal Function. Prostaglandins \& Other Lipid Mediators, 77, 52-64. https://doi.org/10.1016/j.prostaglandins.2004.09.005

[7] Wang, G., Volkow, N., Logan, J., et al. (2001) Brain Dopamine and Obesity. The Lancet, 357, 354-357. https://doi.org/10.1016/S0140-6736(00)03643-6

[8] Volkow, N.D. and O’Brien, C.P. (2007) Issues for DSM-V: Should Obesity Be In- 
cluded as a Brain Disorder? American Journal of Psychiatry, 164, 708-710. https://doi.org/10.1176/ajp.2007.164.5.708

[9] Ward, M.A., Carlsson, C.M., Trivedi, M.A., Sager, M. and Johnson, S. (2005) The Effect of Body Mass Index on Global Brain Volume in Middle-Aged Adults: A Cross Sectional Study. BMC Neurology, 5, 23.

https://doi.org/10.1186/1471-2377-5-23

[10] Gustafson, D., Lissner, L., Bengtsson, C., Björkelund, C. and Skoog, I. (2004) A 24-Year Follow-up of Body Mass Index and Cerebral Atrophy. Neurology, 63, 1876-1881. https://doi.org/10.1212/01.WNL.0000141850.47773.5F

[11] Dixon, J.B., Dixon, M.E. and O’Brien, P.E. (2003) Depression in Association with Severe Obesity. Archives of Internal Medicine, 163, 2058-2065. https://doi.org/10.1001/archinte.163.17.2058

[12] Tariq, A. and Crowther, N. (2005) Health Risks Associated with Obesity. Journal of Endocrinology, Metabolism and Diabetes of South Africa, 10, 56-61.

[13] Mauro, M., Taylor, V., Wharton, S. and Sharma, A.M. (2008) Barriers to Obesity Treatment. European Journal of Internal Medicine, 19, 173-180. https://doi.org/10.1016/j.ejim.2007.09.011

[14] Sarwer, D.B., Fabricatore, A.N., Eisenberg, M.H., Sywulak, L.A. and Wadden, T.A. (2008) Self-Reported Stigmatization among Candidates for Bariatric Surgery. Obesity, 16, S75-S79. https://doi.org/10.1038/oby.2008.450

[15] Kalarchian, M.A., Marcus, M.D., Levine, M.D., et al. (2007) Psychiatric Disorders among Bariatric Surgery Candidates: Relationship to Obesity and Functional Health Status. American Journal of Psychiatry, 164, 328-334. https://doi.org/10.1176/ajp.2007.164.2.328

[16] Faulconbridge, L.F., Wadden, T.A., Berkowitz, R.I., et al. (2009) Changes in Symptoms of Depression with Weight Loss: Results of a Randomized Trial. Obesity, 17, 1009-1016.

[17] Christensen, R., Kristensen, P.K., Bartels, E.M. and Astrup, A. (2007) Efficacy and Safety of the Weight-Loss Drug Rimonabant: A Meta-Analysis of Randomized Trials. The Lancet, 17, 1706-1713. https://doi.org/10.1016/S0140-6736(07)61721-8

[18] Kolotkin, R.L., Corey-Lisle, P.K., Crosby, R.D., et al. (2008) Impact of Obesity on Health-Related Quality of Life in Schizophrenia and Bipolar Disorder. Obesity, 16, 749-754. https://doi.org/10.1038/oby.2007.133

[19] Simon, G.E., Korff, M.V., Sanders, K., Migioretti, D.L., Crane, P.K., Belle, G.V. and Kessler, R.C. (2006) Association between Obesity and Psychiatric Disorders in the US Population. Archives of General Psychiatry, 63, 824-830.

[20] Wang, G.J., Volkow, N.D. and Thanos, P.K. (2004) Similarity between Obesity and Drug Addiction as Assessed by Neurofunctional Imaging: A Concept Review. Journal of Addictive Diseases, 23, 39-53.

[21] Sarwer, D.B., Fabricatore, A.N., Corneille, L.J., et al. (2008) Psychosocial Issues Following Bariatric Surgery. Primary Psychiatry, 15, 50-55.

[22] Onyike, C.U., Crum, R.M., Lee, H.B., Lyketsos, C.G. and Eaton, W.W. (2003) Is Obesity Associated with Major Depression? Results from the Third National Health and Nutrition Examination Survey. American Journal of Epidemiology, 158, 1139-1147. https://doi.org/10.1093/aje/kwg275

[23] Carpenter, K.M., Hasin, D.S., Allison, D.B. and Faith, M.S. (2000) Relationships between Obesity and DSM-IV Major Depressive Disorder, Suicide Ideation, and Suicide Attempts: Results from a General Population Study. American Journal of 
Public Health, 90, 251-257. https://doi.org/10.2105/AJPH.90.2.251

[24] Luppino, F.S., de Wit, L.M., Bouvy, P.F., Stijnen, T., Cuijpers, P., Penninx, B.W. and Zitman, F.G. (2010) Overweight, Obesity, and Depression: A Systematic Review and Meta-Analysis of Longitudinal Studies. Archives of General Psychiatry, 67, 220-229. https://doi.org/10.1001/archgenpsychiatry.2010.2

[25] Zeller, M.H., Roehrig, H.R., Modi, A.C., Daniels, S.R. and Inge, T.H. (2006) Health-Related Quality of Life and Depressive Symptoms in Adolescents with Extreme Obesity Presenting for Bariatric Surgery. Pediatrics, 117, 1155-1161. https://doi.org/10.1542/peds.2005-1141

[26] Allison, K.C., Wadden, T.A., Sarwer, D.B., et al. (2007) Night Eating Syndrome and Binge Eating Disorder among Persons Seeking Bariatric Surgery: Prevalence and Related Features. Obesity, 15, 1287-1293.

[27] Gadalla, T.M. (2009) Association of Obesity with Mood and Anxiety Disorders in the Adult General Population. Chronic Diseases in Canada, 30, 29-36.

[28] Grilo, C.M., White, M.A., Barnes, R.D. and Masheb, R.M. (2013) Psychiatric Disorder Co-Morbidity and Correlates in an Ethnically Diverse Sample of Obese Patients with Binge Eating Disorder in Primary Care Settings. Comprehensive Psychiatry, 54, 209-216.

[29] Jorm, A.F., Korten, A.E., Christensen, H., Jacomb, P.A., Rodgers, B. and Parslow, R.A. (2003) Association of Obesity with Anxiety, Depression and Emotional Well-Being: A Community Survey. Australian and New Zealand Journal of Public Health, 27, 434-440. https://doi.org/10.1111/j.1467-842X.2003.tb00423.x

[30] Lykouras, L. and Michopoulos, J. (2011) Anxiety Disorders and Obesity. Psychiatrike, 22, 307-313.

[31] Martinho, F.L., Tangerina, R.P., Moura, S.M.G.T., Gregório, L.C., Tufik, S. and Bittencourt, L.R.A. (2008) Systematic Head and Neck Physical Examination as a Predictor of Obstructive Sleep Apnea in Class III Obese Patients. Brazilian Journal of Medical and Biological Research, 41, 1093-1097. https://doi.org/10.1590/S0100-879X2008001200008

[32] Pannain, S. and Mokhlesi, B. (2010) Bariatric Surgery and Its Impact on Sleep Architecture, Sleep-Disordered Breathing, and Metabolism. Best Practice \& Research. Clinical Endocrinology \& Metabolism, 24, 745-761.

[33] Benetó, A., Gomez-Siurana, E. and Rubio-Sanchez, P. (2009) Comorbidity between Sleep Apnea and Insomnia. Sleep Medicine Reviews, 13, 287-293.

https://doi.org/10.1016/j.smrv.2008.09.006

[34] Haines, K., Nelson, L.G. and Gonzalez, R. (2007) Objective Evidence that Bariatric Surgery Improves Obesity-Related Obstructive Sleep Apnea. Surgery, 141, 354-358. https://doi.org/10.1016/j.surg.2006.08.012

[35] Bhattacharjee, R., Kheirandish-Gozal, L., Pillar, G. and Gozal, D. (2009) Cardiovascular Complications of Obstructive Sleep Apnea Syndrome: Evidence from Children. Progress in Cardiovascular Diseases, 51, 416-433. https://doi.org/10.1016/j.pcad.2008.03.002

[36] Lopez, P.P., Stefan, B., Schulman, C.I. and Byers, P.M. (2008) Prevalence of Sleep Apnea in Morbidly Obese Patients Who Presented for Weight Loss Surgery Evaluation: More Evidence for Routine Screening for Obstructive Sleep Apnea before Weight Loss Surgery. The American Journal of Surgery, 74, 834-838.

[37] Hallowell, P.T., Stellato, T.A., Schuster, M., et al. (2007) Potentially Life-Threatening Sleep Apnea Is Unrecognized without Aggressive Evaluation. The American Journal of Surgery, 193, 364-367. 
https://doi.org/10.1016/j.amjsurg.2006.09.022

[38] Reynolds, C.F., Kupfer, D.J., McEachran, A.B., Taska, L.S., Sewitch, D.E. and Coble, P.A. (1984) Depressive Psychopathology in Male Sleep Apneics. The Journal of Clinical Psychiatry, 45, 287-290.

[39] Buchwald, H., Avidor, Y., Braunwald, E., et al. (2004) Bariatric Surgery: A Systematic Review and Meta-Analysis. The Journal of the American Medical Association, 292, 1724-1736. https://doi.org/10.1001/jama.292.14.1724

[40] Fritscher, L.G., Canani, S., Mottin, C.C., et al. (2007) Bariatric Surgery in the Treatment of Obstructive Sleep Apnea in Morbidly Obese Patients. Respiration, 74, 647-652. https://doi.org/10.1159/000107736

[41] Hays, H.P. and Roberts, S.B. (2008) Aspects of Eating Behaviors 'Disinhibition' and 'Restraint' Are Related to Weight Gain and BMI in Women. Obesity, 16, 52-58. https://doi.org/10.1038/oby.2007.12

[42] Sarwer, D.B. and Fabricatore, A.N. (2008) Psychiatric Considerations of the Massive Weight Loss Patient. Clinics in Plastic Surgery, 35, 1-10. https://doi.org/10.1016/j.cps.2007.08.006

[43] Friedman, K.E., Reichmann, S.K., Costanzo, P.R., et al. (2005) Weight Stigmatization and Ideological Beliefs: Relation to Psychological Functioning in Obese Adults. Obesity Research, 13, 907-916. https://doi.org/10.1038/oby.2005.105

[44] Friedman, K.E., Ashmore, J.A. and Applegate, K.L. (2008) Recent Experience of Weight-Based Stigmatization in a Weight Loss Surgery Population: Psychoogical and Behavioral Correlates. Obesity, 16, S69-S74.

[45] Carles, R.A., Young, K.M., Wott, C.B., et al. (2009) Internalized Weight Stigma and Its Ideological Correlates among Weight Loss Treatment Seeking Adults. Eating and Weight Disorders-Studies on Anorexia, Bulimia and Obesity, 14, 92-97. https://doi.org/10.1007/BF03327805

[46] Villela, N.B., Neto, O.B., Curvello, K.L., et al. (2004) Quality of Life of Obese Patients Submitted to Bariatric Surgery. Nutrición Hospitalaria, 6, 367-371.

[47] Wille, N., Bullinger, M. and Holl, R. (2010) Health-Relate Quality of Life in Overweight and Obese Youths: Results of a Multicenter Study. Health and Quality of Life Outcomes, 8, 36. https://doi.org/10.1186/1477-7525-8-36

[48] Zeller, M.H. and Modi, A.C. (2006) Predictors of Health-Related Quality of Life in Obese Youth. Obesity, 14, 122-130. https://doi.org/10.1038/oby.2006.15

[49] Fielding, C.R. and Fielding, G. (2008) The Obesity Epidemics, Bariatric Surgery and the Role of the Practitioner. Primary Psychiatry, 15, 40-41.

[50] Bond, D.S., Phelan, S., Wolfe, L.G., et al. (2009) Becoming Physically Active after Bariatric Surgery Is Associated with Improved Weight Loss and Health-Related Quality of Life. Obesity, 17, 78-83. https://doi.org/10.1038/oby.2008.501

[51] Dymek, M.B., le Grange, D., Neven, K. and Alverdy, J. (2002) Quality of Life after Gastric Bypass Surgery: A Cross-Sectional Study. Obesity Research, 10, 1135-1142. https://doi.org/10.1038/oby.2002.154

[52] Busetto, L., Segato, G., De Luca, M., et al. (2005) Weight Loss and Postoperative Complications in Morbidly Obese Patients with Binge Eating Disorder Treated by Laparoscopic Adjustable Gastric Banding. Obesity Surgery, 15, 195-201. https://doi.org/10.1381/0960892053268327

[53] Niego, S.H., Kofman, M.D., Weiss, J.J. and Geliebeter, A. (2007) Binge Eating in the Bariatric Surgery Population: A Review of the Literature. International Journal of Eating Disorders, 40, 349-359. https://doi.org/10.1002/eat.20376 
[54] Andersen, J.R., Aasprang, A., Bergsholm, P., et al. (2010) Anxiety and Depression in Association with Morbid Obesity: Changes with Improved Physical Health after Duodenal Switch. Health and Quality of Life Outcomes, 8, 52. https://doi.org/10.1186/1477-7525-8-52

[55] Jiang, W., Gagliardi, J.P., Raj, Y.P., et al. (2006) Acute Psychotic Disorder after Gastric Bypass Surgery: Differential Diagnosis and Treatment. American Journal of Psychiatry, 163, 15-19. https://doi.org/10.1176/appi.ajp.163.1.15

[56] Bond, D.S., Phelan, S., Leahey, T.M., Hill, J.O. and Wing, R.R. (2009) Weight Loss Maintenance in Successful Weight Losers: Surgical versus Non-Surgical Methods. International Journal of Obesity, 33, 173-180.

[57] Snow, V., Barry, P., Fitterman, N., Qaseem, A. and Weiss, K. (2005) Pharmacologic and Surgical Management of Obesity in Primary Care: A Clinical Practice Guideline from the American College of Physicians. Annals of Internal Medicine, 142, 525-531. https://doi.org/10.7326/0003-4819-142-7-200504050-00011

[58] Li, Z., Maglione, M., Tu, W., et al. (2005) Meta-Analysis: Pharmacologic Treatment of Obesity. Annals of Internal Medicine, 142, 532-546. https://doi.org/10.7326/0003-4819-142-7-200504050-00012

[59] Reever, M.M. (2008) Cognitive-Behavioral Interventions for Obesity. Northeast Florida Medicine, 59, 37-40.

[60] Levy, R.L., Finch, E.A., Crowell, M.D. and Talley, N.J. (2007) Behavioral Intervention for the Treatment of Obesity: Strategies and Effectiveness Data. The American Journal of Gastroenterology, 102, 2314-2321.

[61] Blaine, B. (2008) Does Depression Cause Obesity? A Meta-Analysis of Longitudinal Studies of Depression and Weight Control. Journal of Health Psychology, 13, 1190-1197.

[62] Chaput, J.P., Klingenberg, L., Rosenkilde, M., et al. (2011) Physical Activity Plays an Important Role in Body Weight Regulation. Journal of Obesity, 2011, Article ID: 360257. https://doi.org/10.1155/2011/360257

[63] Yumuk, V., Tsigos, C., Fried, M., et al. (2015) European Guidelines for Obesity Management in Adults. Obesity Facts, 8, 402-424. https://doi.org/10.1159/000442721

[64] Fabricatore, A.N., Wadden, T.A., Higginbotham, A.J., et al. (2011) Intentional Weight Loss and Changes in Symptoms of Depression: A Systematic Review and Meta-Analysis. International Journal of Obesity, 35, 1363-1376. https://doi.org/10.1038/ijo.2011.2

[65] Bartholomew, J.B., Morrison, D. and Ciccolo, J.T. (2005) Effects of Acute Exercise on Mood and Well-Being in Patients with Major Depressive Disorder. Medicine \& Science in Sports \& Exercise, 37, 2032-2037. https://doi.org/10.1249/01.mss.0000178101.78322.dd

[66] Craft, L.L. and Perna, F.N. (2004) The Benefits of Exercise for the Clinically Depressed. The Primary Care Companion to The Journal of Clinical Psychiatry, 6, 104-111.

[67] Cooper, Z., Doll, H.A., Hawker, D.M., Byrne, S., Bonner, G., Eeley, E., O’Connor, M.E. and Fairburn, C.G. (2010) Testing a New Cognitive Behavioral Trial with Three-Year Follow-Up. Behaviour Research and Therapy, 48, 706-713. https://doi.org/10.1016/j.brat.2010.03.008 